\title{
Simultaneous Determination of the Pharmacokinetics and Pharmacodynamics of Chlorpromazine in the Brain of Mice ${ }^{\dagger}$
}

\author{
Yukinobu GOTOH, Shinji SHIBANOKI and Koichi ISHIKAWA \\ Department of Pharmacology. Nihon University School of Medicine, \\ Itabashi, Tokyo 173, Japan \\ Accepted October 14, 1985
}

\begin{abstract}
The direct correlation analyses between the distribution of chlorpromazine (pharmacokinetics) and the biochemical effects of the drug on monoamine metabolisms (pharmacodynamics) are reported. Both samples for quantitative determination of $\mathrm{CPZ}$ and of monoamine transmitters and metabolites were obtained by organic extraction procedures from the same sample. The determinations were carried out by high performance liquid chromatography with electrochemical detection. CPZ affected the concentrations of metabolites of noradrenaline, dopamine and 5 -hydroxytryptamine, but not those of the monoamine transmitters themselves. However, simultaneous assay demonstrated differences in effects of the drug on the transmitter systems. The concentrations of HVA and DOPAC were increased over a wide range of intracerebral concentrations of the drug, but those of MOPEG, in the range of higher concentrations. On the other hand, CPZ did not reveal any correlations between the intracerebral concentrations of the drug and 5-HIAA. These results suggest that CPZ affected primarily the dopaminergic system rather than the serotonergic one in the early stage of its biochemical actions. The proposed procedure is demonstrated to be simple and useful as a new approach in biochemical pharmacology. The same procedure can be applicable for other centrally acting drugs.
\end{abstract}

Pharmacological action can be expressed as a function of the concentration of a drug at the site of action. This means that, where possible, in vivo correlations between the intracerebral concentration of a drug and the effect induced by the drug provide important information on the pharmacology of CNS acting drugs. The intracerebral concentration varies according to pharmacokinetic factors, which cannot be predicted because of individual differences. Thus, simultaneous determinations of the pharmacokinetics and pharmacodynamics of a drug are useful in pharmacological research. In a previous report, we described such determinations for morphine (1).

The phenothiazine derivatives are drugs

t Part of this study was presented at the 9th International Congress of Pharmacology, London. England. August, 1984 used in the treatment of psychotic disorders and they display complex pharmacological profiles (2-4). For example, the phenothiazines have been reported to share antiadrenergic, anti-cholinergic and antihistaminergic properties. Some of the recent reviews have emphasized that the phenothiazines are strong dopamine receptor blockers (2, 3) and are useful tools for studying the dopaminergic involvement in functions of the central nervous system. If simultaneous determinations of the distribution and biochemical effects on monoamine transmitters were possible, the detailed profile of the mode of action of phenothiazines might be elucidated.

Electrochemical detection (ECD) have recently been introduced in the chromatographic assay of monoamine related substances $(5-10)$. The procedure has also been shown to be applicable for the assay of 
chlorpromazine in the brain and blood (11). This suggests that ECD is suitable for use in the simultaneous determination of the pharmacokinetics and pharmacodynamics of CPZ, the prototype of phenothiazine antipsychotics. The present study was therefore undertaken in an attempt to carry out simultaneous determinations of CPZ and substances metabolically related to monoamine transmitters in the brain of mice.

\section{Materials and Methods}

Animals: ICR mice each weighing about $30 \mathrm{~g}$ were used throughout the experiments. The animals were housed in a cage in a quiet room with a controlled temperature $\left(23 \pm 1^{\circ} \mathrm{C}\right)$, humidity $(55 \pm 5 \%)$ and light cycle (12 hr light/12 hr dark) with the lights being turned on at 07:00 hr. They were given free access to food and water. Prior to sacrifice, all animals were maintained in the room for a minimum of one week to exclude the influence of habitat stress. The sacrifice time was fixed between 13:00 to $15: 00 \mathrm{hr}$ in order to minimize the effects of circadian rhythm on biogenic amine metabolism.

Chlorpromazine hydrochloride (CPZ: Shionogi Pharmaceuticals, Osaka, Japan) was injected intravenously into animals in the dose range between 0.1 and $20 \mathrm{mg} / \mathrm{kg}$. In the first series of experiments, time course changes in the concentrations of monoamine related substances were studied for the drug. The animals were intravenously injected with $10 \mathrm{mg} / \mathrm{kg}$ of $\mathrm{CPZ}$ and were sacrificed at different times after the injection. Another experiment was carried out to demonstrate the direct correlation between the intracerebral concentrations of CPZ and monoamine metabolites, where the animals were sacrificed by decapitation at $30 \mathrm{~min}$ after the injection of different doses of the drug.

The brain and blood were obtained as quickly as possible after sacrifice. The brain sample was weighed and then wrapped in aluminium foil for storage in a deep freezer $\left(-80^{\circ} \mathrm{C}\right)$ until assay. The blood sample was collected from the carotid artery when the animal was decacitated. The whole blood was also stored in the deep freezer.

Apparatus: Liquid chromatography was performed using a Yanagimoto L-2000 pump (Yanagimoto, Kyoto, Japan), a Rheodyne 7125 six port injector (Rheodyne, Berkeley. CA, U.S.A.) and an Ultrasphere ODS reverse-phase column $(250 \mathrm{~mm} \times 4.6 \mathrm{~mm}$ I.D., average particle size $5 \mu . n$; Altex Scientific, Berkeley, CA, U.S.A.). To protect the analytical column, a short column (10 $\mathrm{mm} \times 4.5 \mathrm{~mm}$ I.D.) with ODS particles was installed. The electrochemical detector (Yanagimoto, VMD-501) worked at applied voltages of $600,700,600$ and $800 \mathrm{mV}$ versus the $\mathrm{Ag} / \mathrm{AgCl}$ reference electrode for amine, acidic metabolite, MOPEG and CPZ assays, respectively.

Chemicals: Authentic standard substances for the present assay, including noradrenaline $\mathrm{HCl}$ (NA), dopamine $\mathrm{HCl}$ (DA), 5-hydroxytryptamine $\mathrm{HCl} \quad(5-\mathrm{HT})$, 3-methoxy-4hydroxyphenylethylene glycol piperidine salt (MOPEG), 3,4-dihydroxyphenylacetic acid (DOPAC), homovanillic acid (HVA) and 5hydroxyindoleacetic acid (5-HIAA), were all purchased from Sigma (St. Louis, MO, U.S.A.). The internal standards, isoproterenol $\mathrm{HCl}$ (ISO, for amine and MOPEG assays). 3,4-dihydroxyphenylpropionic acid (DOPPA, for acidic metabolite assay) and promethazine $\mathrm{HCl}(\mathrm{PMZ})$, were obtained from Aldrich (Milwaukee, WI, U.S.A.), ICN Pharmaceuticals (Plainview, NY, U.S.A.) and Shionogi Pharmaceuticals, respectively. Each substance was separately dissolved in $0.1 \mathrm{~N} \mathrm{HCl}$ at a concentration of $1.0 \mathrm{mg} / \mathrm{ml}$ and stored in a refrigerator. The standard substances were freshly prepared each month. The working standard solution was made up on the day of assay from the stock solution. Reagent grade chemicals for extraction and chromatography were obtained from a single source (Wako Pure Chemicals, Osaka, Japan) and used without further purification.

Extraction procedure: The brain tissue was homogenized by a Polytron (Kinematica, Luzern, Switzerland) in a glass stoppered tube containing $1 \mathrm{ml}$ of $0.025 \mathrm{~N} \mathrm{HCl}$ and internal standards (ISO, DOPPA and PMZ). Five hundred $\mu l$ of the homogenate was transferred to another glass stoppered tube for CPZ assay, for which the extraction was performed as described previously (11). In 
brief, $500 \mu \mathrm{l}$ of $1 \mathrm{~N} \mathrm{NaOH}$ and $5 \mathrm{ml}$ of an organic mixture of heptane : isoamyl alcohol (99:1) were added to the tube. The mixture was then vortexed for $1 \mathrm{~min}$ and centrifuged for $3 \mathrm{~min}$ to separate the organic layer, of which $4 \mathrm{ml}$ was transferred to another tube. After adding $100 \mu l$ of $0.1 \mathrm{~N} \mathrm{HCl}$, the tube was vortexed for $1 \mathrm{~min}$. The $\mathrm{HCl}$ layer resulting from centrifugation was used as the chromatographic sample.

The remaining initial homogenate was centrifuged at $1,000 \mathrm{~g}$ for $30 \mathrm{~min}$. A portion $(100 \mu l)$ of the supernatant was transferred to a polyethylene tube and $200 \mu \mathrm{l}$ of $0.2 \mathrm{M}$ acetate buffer ( $\mathrm{pH}$ 5.0) containing sulfatase (12.5 units $/ \mathrm{ml}$ ) was added. The polyethylene tube was incubated at $37^{\circ} \mathrm{C}$ for $12 \mathrm{hr}$ and then filtrated with a Millipore filter (pore size. $0.45 \mu \mathrm{m})$. The filtrate was stored in a refrigerator at $4^{\circ} \mathrm{C}$ until MOPEG assay (10). The residual homogenate was treated by a procedure reported previously for monoamine transmitters and their precursors and acidic metabolites (9). Briefly, solid sodium chloride, EDTA and $n$-butanol were added to the homogenate. The tube was shaken on a reciprocal shaker for $60 \mathrm{~min}$ and then centrifuged at $1.000 \mathrm{~g}$ for $10 \mathrm{~min}$. The butanol layer was transferred to another tube containing $0.1 \mathrm{~N} \mathrm{HCl}$ and $n$-heptane. The tube was shaken again for $10 \mathrm{~min}$ and then centrifuged at $1,000 \mathrm{~g}$ for $5 \mathrm{~min}$. A portion of the $\mathrm{HCl}$ layer was injected into the HPLC system. The organic layer was transferred to a fresh glass tube and stored in a refrigerator at $4{ }^{\circ} \mathrm{C}$ until metabolite assay. Tris- $\mathrm{HCl}$ buffer $(\mathrm{pH}$ 8.5) was added to the organic layer stored in the refrigerator. The tube was vortexed for $1 \mathrm{~min}$ and briefly centrifuged to separate organic and aqueous phases. A portion of the aqueous phase was immediately injected into the HPLC system. The working standard for quantitative determination was treated in exactly the same manner as the brain sample.

HPLC analysis: For CPZ assay, the chromatographic mobile phase was a mixture of $0.1 \mathrm{M}$ acetate buffer ( $\mathrm{pH} 3.5$ )-acetonitriletetrahydrofuranpyridine (100:200:3:0.3). For amine and MOPEG assays, $0.1 \mathrm{M}$ sodium citrate-citric acid buffer ( $\mathrm{pH}$ 4.0) containing $1 \%$ tetrahydrofuran (THF) was used at a flow rate of $1.3 \mathrm{ml} / \mathrm{min}$. On the other hand, 0.075 $M$ sodium citrate-citric acid buffer ( $\mathrm{pH} 3.5$ ) containing $1 \%$ THF, $10 \%$ methanol and $12 \%$ acetic acid was employed at a flow rate of $0.8 \mathrm{ml} / \mathrm{min}$ for metabolite assay (in which DOPAC, HVA and 5-HIAA were determined). In the case of MOPEG assay, the buffer for metabolite assay was used to wash out biogenic substances with a long retention time after the MOPEG peak was recorded. These mobile phases were sonicated under vacuum in order to eliminate air bubbles, which tend to interfere with the electrochemical detection. The temperature of the analytical column was controlled at $35^{\circ} \mathrm{C}$ with a water jacket.

Calculations: The content of each substance was determined by comparison of the peak heights in the sample and standard. Working standards were run at regular intervals to check the sensitivity of the detector. The concentration of each substance was calculated by correcting for recovery.

\section{Results}

In the CPZ assay, no biogenic substance interfered with the quantitative determination in the brain as well as the blood when the detector voltage was set at $800 \mathrm{mV}$. The retention time of the drug was $5.2 \mathrm{~min}$, and one chromatographic run was completed within $7 \mathrm{~min}$. The detection limit based on an $S / N$ ratio of $10: 1$ was $1 \mathrm{ng}$. The variation on repeated injection of the same amount of standard was less than 3\%. The accuracy of the other assays including monoamines, MOPEG and acidic metabolites was also established. The optimal applied voltages were decided at 600,700 and $600 \mathrm{mV}$ for the assays of monoamines, MOPEG and acidic metabolites, respectively. These voltages did not cause interfering peaks due to biogenic substances. The assay of monoamine transmitters (including NA, DA and 5-HT) was completed within $11 \mathrm{~min}$. In the case of MOPEG or acidic metabolite assay, the run time of one chromatography was 9 or $7 \mathrm{~min}$, respectively. The detection limit for each substance was $200 \mathrm{pg}$. In the present assay, amino acids were excluded because of no pharmacological significance. If determination 
of the precursor amino acids, tyrosine and tryptophan, is necessarily involved, the applied voltage should be set at $800 \mathrm{mV}$.

The pharmacokinetics of intracerebral CPZ were assessed after the intravenous injection of different doses (Fig. 1). The brain levels of the drug were dependent on the dose injected, showing values of $20.8 \pm 3.5$ (mean \pm S.D. of 5 determinations), $9.8 \pm 2.6$ and $2.4 \pm 0.6 \mu \mathrm{g} / \mathrm{g}$ wet tissue at $30 \mathrm{~min}$ (when the maximum concentrations were observed) after the injection of 20, 10 and $2.5 \mathrm{mg} / \mathrm{kg} \mathrm{CPZ}$, respectively. Thereafter, the levels declined linearly with significant correlation coefficients over -0.99 . The time course curves of the three doses were arranged in a parallel fashion, and the biological half life was estimated to be about $65 \mathrm{~min}$ without difference among the doses. These data suggested that CPZ passively penetrated into the brain according to its lipid/blood distribution coefficient. The half life in the blood was also estimated at $87 \mathrm{~min}$ in the case of the $10 \mathrm{mg} / \mathrm{kg}$ injection. Only about $2 \%$ of the initial level (theoretical levels at $0 \mathrm{~min}$ ) remained in the brain at $6 \mathrm{hr}$ after the injection, when the final sample was obtained.

The time courses of the changes in concentrations of monoamine transmitters after

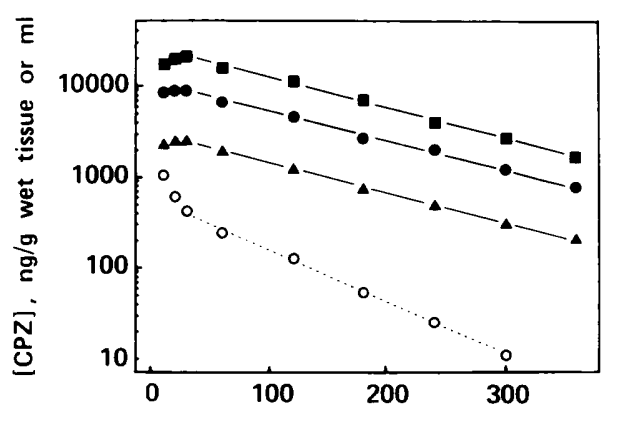

Time after injection ( $\mathrm{min})$

Fig. 1. Elimination of CPZ from the brain and blood of mice. For intracerebral analyses, CPZ was intravenously injected at dose levels of 20 ( $\mathbf{D}) .10$ (O) and $2.5 \mathrm{mg} / \mathrm{kg}(\boldsymbol{\Delta})$, and then the samples were obtained at different times. The blood samples ( $O$ ) were also obtained from the animals injected with $10 \mathrm{mg} / \mathrm{kg}$ of the drug. Note that the intracerebral concentrations of the drug were always higher than those in the blood including the earlier stages ( $5 \mathrm{~min}$ ). intravenous injection of CPZ (10 $\mathrm{mg} / \mathrm{kg})$ were examined (conventional analysis). Neither NA nor DA showed any significant change in intracerebral concentration at any time after the injection (Fig. 2). The indoleamine was also unchanged by the intravenous injection of $\mathrm{CPZ}$.

On the other hand, the monoamine metabolites (MOPEG, DOPAC, HVA and 5-HIAA) were significantly increased after intravenous injection of $10 \mathrm{mg} / \mathrm{kg}$ of $\mathrm{CPZ}$ (Fig. 3). The concentration of MOPEG in control animals was $100 \pm 25 \mathrm{ng} / \mathrm{g}$ wet tissue (mean \pm S.D. of 5 determinations). The maximum increase was observed at $1 \mathrm{hr}$ after injection $(195 \pm 21 \mathrm{ng} / \mathrm{g})$. In the case of DOPAC, the concentration was increased from a control value of $107 \pm 13$ to $281 \pm 51$ $\mathrm{ng} / \mathrm{g}$ at $2 \mathrm{hr}$ after the injection, i.e., about 2.8-fold with respect to the control. HVA, another DA metabolite, was increased 2.7fold with respect to the control value at $2 \mathrm{hr}$ after the injection. 5-HIAA was also sig-

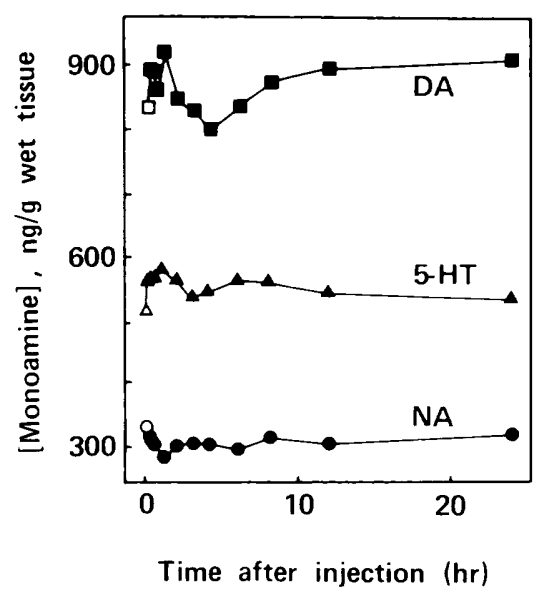

Fig. 2. Effects of $\mathrm{CPZ}$ on the concentration of monoamine transmitters in the brain (conventional analysis). Animals were sacrificed at different times after intravenous injection of $10 \mathrm{mg} / \mathrm{kg}$ of CPZ. NA (O), DA ( $\mathbf{\square})$ and 5-HT (A) were determined simultaneously by a combination of the organic extraction and HPLC-ECD. Each point represents the mean of 10 determinations. The control values (means, shown by corresponding open marks) were 337.983 and $524 \mathrm{ng} / \mathrm{g}$ wet tissue for NA, DA and $5-\mathrm{HT}$, respectively. No significant changes in monoamine levels were observed at any time in comparison with those of the control. 
nificantly increased when the determination was carried out $2 \mathrm{hr}$ after the intravenous injection of $10 \mathrm{mg} / \mathrm{kg} \mathrm{CPZ}$. Thus, CPZ induced significant changes in the concen-

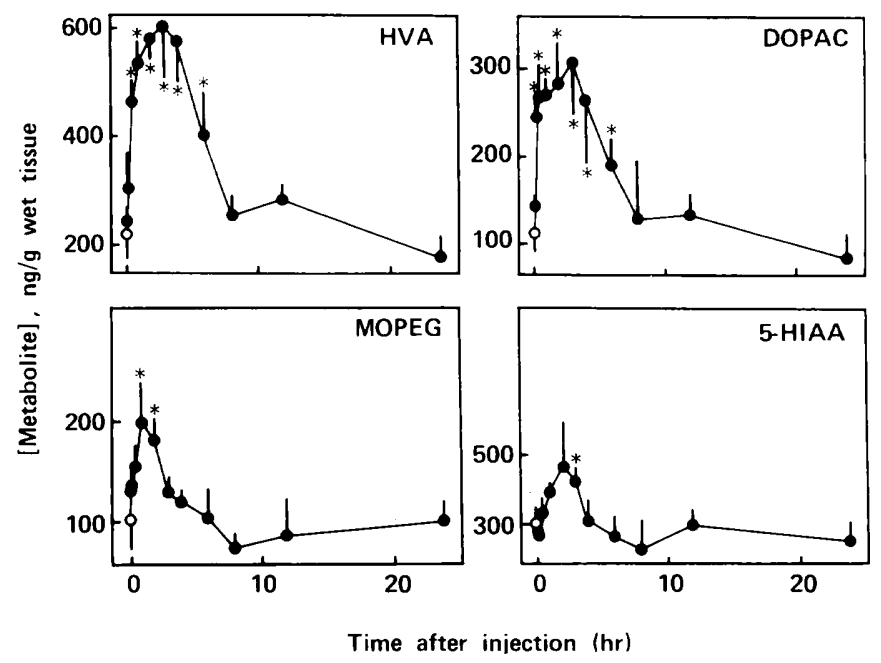

Fig. 3. Effects of CPZ on the concentration of monoamine metabolites in the brain (conventional analysis). Each point with bar represents the mean \pm S.D. of 10 determinations. The control values (C, mean \pm S.D.) were $210 \pm 25,107 \pm 13,100 \pm 25$ and $284 \pm 12 \mathrm{ng} / \mathrm{g}$ wet tissue for HVA, DOPAC, MOPEG and 5-HIAA, respectively. The asterisks indicate a significant difference from the control. Note that all monoamine metabolites were significantly affected by the administration of CPZ.

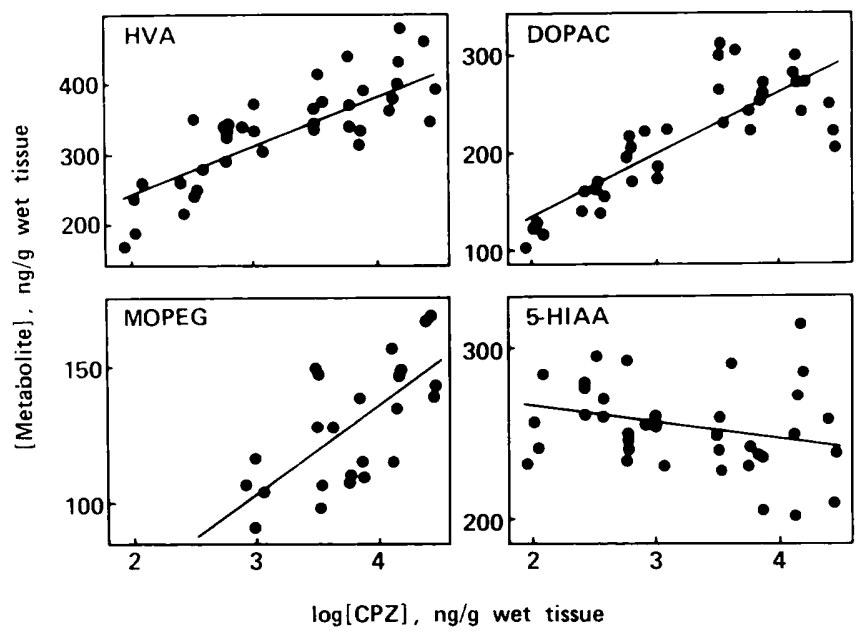

Fig. 4. Correlation between intracerebral concentrations of $C P Z$ and monoamine metabolites. Animals were injected with different doses of the drug and sacrificed at $30 \mathrm{~min}$ after the injection. The concentrations of $\mathrm{CPZ}$ and metabolites were measured in the same sample of the mouse brain. The regression coefficients were estimated at 0.756 and 0.802 for HVA and DOPAC, respectively, in the range between 94 and $29,083 \mathrm{ng} / \mathrm{g}$ wet tissue of the drug. These values were statistically significant $(P<0.001)$. In the case of MOPEG, the regression coefficient $(0.746)$ was significantly $(P<0.001)$ correlated in the higher range over $818 \mathrm{ng} / \mathrm{g}$ wet tissue of the drug. On the other hand, the regression coefficient of 5-HIAA (-0.164) was not significant in any range of intracerebral concentrations of the drug. 
trations of monoamine metabolites, but the potencies differed from one to the other.

The direct correlations between the intracerebral concentrations of CPZ and monoamine metabolites were examined (Fig. 4). The maximal incremental effect of CPZ on monoamine metabolites reached a plateau at an intracerebral concentration of $29.083 \mathrm{ng} / \mathrm{g}$ wet tissue: higher levels of intracerebral CPZ did not reveal linear increase in monoamine metabolite levels. Thus, the effects of higher levels (greater than 30,000 $\mathrm{ng} / \mathrm{g}$ ) of CPZ were excluded from the present correlation analyses. The intracerebral levels of DOPAC $(r=0.802, N=37)$ and HVA $(r=0.756, N=37)$ were significantly correlated $(P<0.001)$ with the $C P Z$ levels in the range from 94 to $29.083 \mathrm{ng} / \mathrm{g}$ wet tissue in animals sacrificed at $30 \mathrm{~min}$ after injection of the drug. On the other hand, the regression coefficient between the concentrations of CPZ and 5-HIAA was lower $(r=-0.164$, $N=37, P>0.1)$ than those for the dopaminergic system. In the case of MOPEG, the correlation was significant $(r=0.746$, $N=22, P<0.001$ ) in the higher range of concentrations of the drug from 818 to $29.083 \mathrm{ng} / \mathrm{g}$ wet tissue.

\section{Discussion}

Previous studies have demonstrated biochemical effects of CPZ on the metabolism of monoamine transmitters (12-14). These studies indicated changes in the concentrations of the transmitters as well as their metabolites in proportion to the injected dose. The intracerebral concentrations of the drug are influenced by pharmacokinetic factors of the animal. The blood-brain barrier restricts the penetration of drugs into the brain. The action of centrally acting drugs therefore fluctuates significantly if the action is determined in relation to the dose administered. The biochemical effect of a drug should ideally be measured as a function of the concentrations of the drug at the site of action. A recent report from this laboratory described the simultaneous determination of morphine and substances metabolically related to monoamine transmitters, and demonstrated the usefulness of such an assay in neuropharmacological studies (1).
ECD has been applied in the determination of substances metabolically related to monoamine transmitters (5-10). The technique was recently employed for the assay of CPZ in the brain (11). It is possible, therefore, to determine simultaneously monoaminerelated substances and $\mathrm{CPZ}$ in the brain by means of HPLC-ECD. Such measurements are expected to provide more precise details of the actions of drugs. The present study was carried out as a model experiment for this purpose.

Other procedures in biochemical studies have indicated that CPZ may act chiefly as a receptor blocking agent of the dopaminergic system, but not of the serotonergic one (15). However, the conventional approach adopted in the present study indicated that the metabolites of not only DA but also NA and 5-HT were affected by intravenous administration of CPZ. These results suggest, if determinations are carried out for each neurotransmission system, that the action of the drug extends to both the catecholaminergic and serotonergic systems. On the other hand, direct correlation analysis indicated that CPZ acted on the dopaminergic system in the brain, whereas the substance did not affect the serotonergic mechanism. The intracerebral concentrations of $\mathrm{CPZ}$ were 94 to $29,083 \mathrm{ng} / \mathrm{g}$ wet tissue when determined $30 \mathrm{~min}$ after the injection at doses of 0.1 (the minimum dose in the present study) and $20 \mathrm{mg} / \mathrm{kg}$ (the maximum). In the range from the lowest to highest levels of the drug, the dopaminergic biochemical parameters, the concentrations of DOPAC and HVA, were significantly correlated to the concentration of $\mathrm{CPZ}$ in the brain. However, the serotonergic parameter, 5-HIAA had no significant correlation coefficient with respect to the intracerebral concentration of the drug. Those findings possibly suggest that the drug primarily affects the dopaminergic mechanism rather than the serotonergic one. In the case of MOPEG, the major metabolite of NA in the brain, a significant correlation was observed in the higher concentration range from 818 to $29,083 \mathrm{ng} / \mathrm{g}$ wet tissue, which was obtained when 1 and $20 \mathrm{mg} / \mathrm{kg}$ of the drug was injected, respectively. This suggests that the noradrenergic system is also affected by the 
injection of CPZ and that the effect emerges with a relatively high concentration at the site of action in comparison with the dopaminergic system. The involvement of the serotonergic mechanism in the direct effect of CPZ may be negated by the present analysis, at least, in the early stage of the actions.

The direct correlation analysis can provide a simple approach for biochemical screening of central acting drugs in vivo. It may be concluded furthermore that $\mathrm{CPZ}$ acted primarily on the dopaminergic transmission system.

Acknowledgement: This work was supported by a Grant-in-Aid for Scientific Research ( \$59480122) from the Japanese Ministry of Education. Science and Culture.

\section{References}

1 Ishikawa, K., Shibanoki, S. and McGaugh, J.L.: Direct correlation between level of morphine and its biochemical effect on monoamine systems in mouse brain. Biochem. Pharmacol. 32, 14731478 (1983)

2 Carlsson, A.: Mechanism of action of neuroleptic drugs. In Psychopharmacology: A Generation of Progress, Edited by Lipton, M.A., DiMascio. A., and Killam, K.F.. p. 1057-1070, Raven Press, New York (1978)

3 Iversen, S.D. and Iversen, L.L.: Behavioral Pharmacology. p. 248-277, Oxford University Press, New York (1981)

4 Teller, D.H.: Phenothiazines and butyrophenones in relation to neurochemistry and pharmacology. In Psychopharmacological Treatment: Theory and Practice, Edited by Denber. H.C.B., p. 1-62, Marcel Dekker, New York (1975)

5 Hegstrand, L.R. and Eichelman, B.: Determination of rat brain tissue catecholamines using liquid chromatography with electrochemical detection. J. Chromatogr. 222, 107-111 (1981)

6 Kempf, E. and Mandel, P.: Reverse-phase high performance liquid chromatography separation and electrochemical detection of norepinephrine, dopamine, serotonin, and related major metabolites. Anal. Biochem. 112, 223-231 (1981)
7 Kissinger, P.T. and Bruntlett, C.S. and Shoup, R.E.: Neurochemical applications of liquid chromatography with electrochemical detection. Life Sci. 28, 455-465 (1981)

8 Wagner, J., Vitali, P., Palfreyman, M.G., Zraika, M. and Huot, S.: Simultaneous determination of 3,4-dihydroxyphenylalanine, 5-hydroxytryptamine, dopamine, 4-hydroxy-3-methoxyphenylalanine, norepinephrine, 3,4-dihydroxyphenylacetic acid, homovanillic acid, serotonin, and 5-hydroxyindole acetic acid in rat cerebrospinal fluid and brain by high-performance liquid chromatography with electrochemical detection. J. Neurochem. 38, 1241-1254 (1982)

9 Ishikawa, K. and McGaugh, J.L.: Simultaneous determination of monoamine transmitters, precursors and metabolites in a single mouse brain. J. Chromatogr. 229, 35-46 (1982)

10 Ishikawa, K. and Shibanoki, S.: Liquid chromatographic determination of free and conjugated 3 methoxy-4-hydroxyphenylethylene glycol with wide-ranging substances related to monoamine metabolism. Anal. Biochem. 147, 441-450 (1985)

11 Shibanoki, S., Gotoh, Y. and Ishikawa, K.: Determination of chlorpromazine in the blood and brain of mice by high performance liquid chromatography combined with electrochemical detection. Japan. J. Pharmacol. 35, 169-174 (1984)

12 Wiesel, F.-A. and Sedvall, G.: Effects of antipsychotic drugs on homovanillic acid levels in striatum and olfactory tubercle of the rat. Eur. J. Pharmacol. 30, 364-367 (1975)

13 Waldmeier, P.C. and Maitre, L.: Clozapine: reduction of the initial dopamine turnover increase by repeated treatment. Eur. J. Pharmacol. 38, 197-203 (1976)

14 Scatton, B., Bischoff, S., Dedek, J. and Korf, J.: Regional effects of neuroleptics on dopamine metabolism and dopamine-sensitive adenylate cyclase activity. Eur. J. Pharmacol. 44, 287-292 (1977)

15 Westerink, B.H.C.: The effects of drugs on dopamine biosynthesis and metabolism in the brain. In The Neurobiology of Dopamine, Edited by Horn, A.S., Korf, J. and Westerink, B.H.C. p. 255-291, Academic Press, London (1979) 